\title{
Compound Radiocarpal Dislocation: A Unique Case Report
}

\author{
S Tontanahal,T.D Bhattacharyya, S Mittal, R Ailani, A Gaikwad \\ Gauhati Medical College, Guwahati, Assam
}

\begin{abstract}
:
Aim: The Aim of this case report is to describe a case of compound radial styloid process fracture with dislocation of the wrist joint and its management.

Case Report: A 55 year male sustained a compound fracture of the radial styloid with dislocation of the wrist joint following a fall from height. This injury was also associated with injury to the tendons of the medial aspect of the wrist joint and the ulnar artery. The patient underwent immediate external fixation and wound closure with no significant complaints at 12 month follow up other than complete ulnar nerve injury.

Discussion: In the present case, the compound fracture dislocation of the wrist joint which was associated with tendon and ulnar artery injury was reduced and fixed immediately on the day of presentation. The results of this management were found to be satisfactory.
\end{abstract}

Conclusion: The case has been reported to highlight the good stability and functional outcome following immediate external fixation of this injury.

Keywords: Radiocarpal dislocation, Compound, Ligamentotaxis, Functional outcomes.

\section{- Introduction}

Radiocarpal dislocation is an uncommon entity in traumatology. Proper management depends on the type of dislocation and the presence of concomitant injury ${ }^{(1)}$. Associated injuries are common with radial styloid, ulnar styloid and marginal avulsion fractures predominating. Open injuries with significant soft-tissue disruption can occur, leading to persistent instability ${ }^{(1)}$. The paucity of reported cases and incomplete understanding of the spectrum of associated injuries has not permitted a consensus on treatment recommendations.

\section{- Case Report}

A 55 year old male sustained a fall from a height of around 5 feet on an out stretched hand at home in April 2015, with impact on the ulnar aspect of the right wrist joint. Following the fall he developed a wound which was around $4 \mathrm{~cm} \times 3 \mathrm{~cm}$. The wound was associated with pain, swelling and deformity around the wrist joint. There was loss of extension of the medial two fingers at the metacarpophalangeal joints. The ulnar artery pulsations were not palpable; however the capillary refill time was normal. The sensations were intact over the cutaneous distribution of the ulnar nerve.

Pre-operative radiographs of the wrist joint including the forearm were obtained in the antero-posterior and lateral views. The $\mathrm{x}$ ray showed a fracture of the radial styloid process and radio-carpal dissociation with a dorsal and lateral displacement. Initial treatment was in the form of wound irrigation, sterile dressing and immobilization with an above elbow POP slab.

After an informed consent, surgery was conducted 6 hours from the time of injury. Following through wash and debridement, the fracture and radio-carpal dissociation were reduced and stabilized with ligamentotaxis with Kirschner wire augmentation. Wound closure was done. Ulnar artery was found transected and the cut ends ligated, which was done as part of primary care outside our centre. There was no attempt at arterial re anastomosis as the capillary refill time was found to be normal. Tendon repair was also not carried out in the same sitting.

Post-operative period was uneventful and the patient was followed up regularly. At 3 weeks following surgery the stitches and Kirschner wires were removed and the wound was dry and had no discharge. The external fixator assembly was removed 6 weeks following surgery. Following removal physiotherapy in the form of active and passive wrist mobilization was initiated.

Twelve months following surgery, the patient was found to have good range of motion at the wrist. Flexion was up to $80^{\circ}$, extension was up to $50^{\circ}$. Supination was restricted by $30^{\circ}$ with no restriction of pronation. However there was complete ulnar nerve injury distal to the wrist which was evident clinically by the presence of ulnar claw hand and wasting of the hypothenar eminence. This was further confirmed by nerve conduction velocity testing of ulnar nerve. The patient was given the option of tendon transfer but the patient party declined this plan of action. 


\section{- Discussion}

Radiocarpal fracture-dislocations are rare injuries, with few reported case series in the English literature $^{(2)}$. Pure radiocarpal dislocation (or in association with a minimal fracture of the styloid) should be treated surgically, irrespective of the approach, in order to achieve capsule-ligament suture ${ }^{(1)}$. Despite improved appreciation for the relevant pathoanatomy of this entity, outcome studies after operative repair of radiocarpal dislocations and fracture-dislocations are not readily available in the literature. Therefore, treatment approaches must be developed that adequately address the spectrum of osseous and soft tissue injuries that occur ${ }^{(3,4,5)}$.

The present case describes a compound fracture dislocation of the radiocarpal joint which was associated with ligament and vascular damage which was treated with immediate external fixation. There was good clinical outcome following surgery.

\section{- Conclusion}

We report a rare case of a compound fracture dislocation of the radiocarpal joint. The immediate surgical stabilization of this resulted in good functional and clinical outcome.

\section{- Figures}
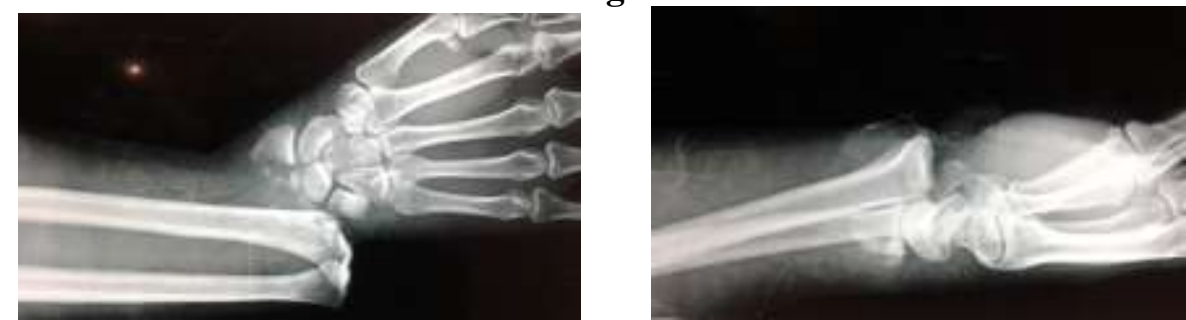

Fig 1. Pre-operative radiographs
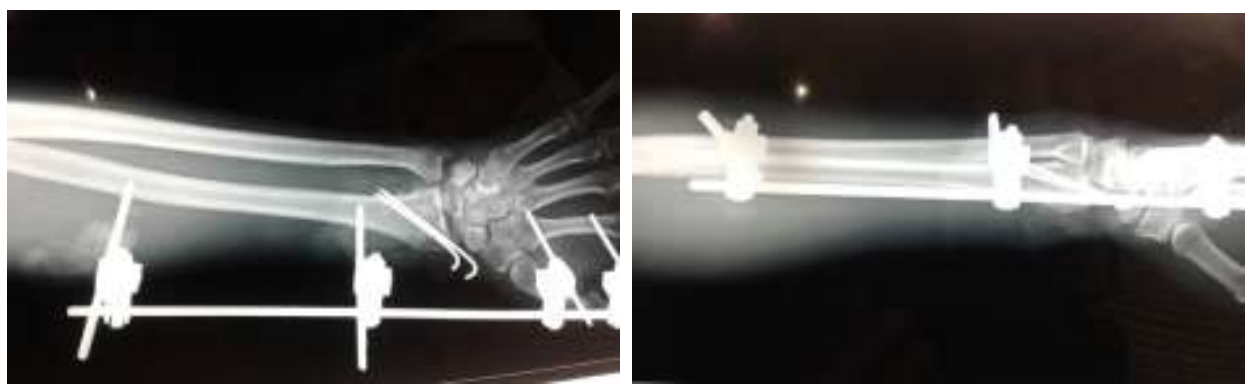

Fig 2. Post-operative radiographs



Fig 3. Pre-operative wound

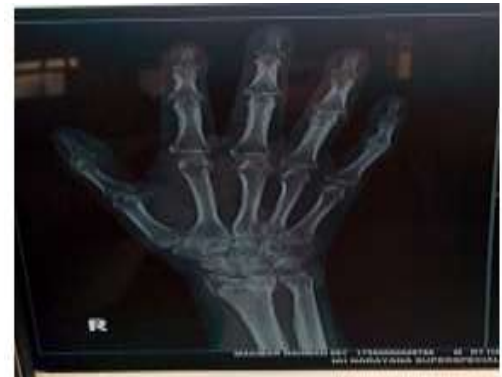

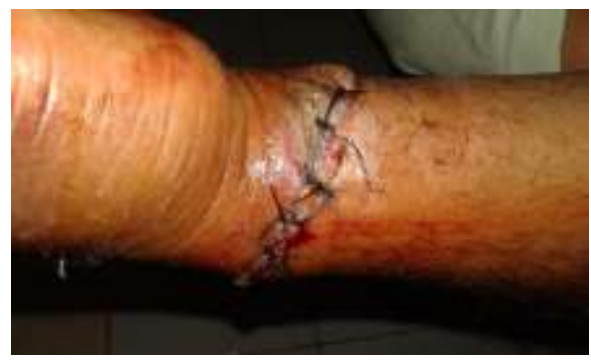

Fig 4. Immediate post-operative

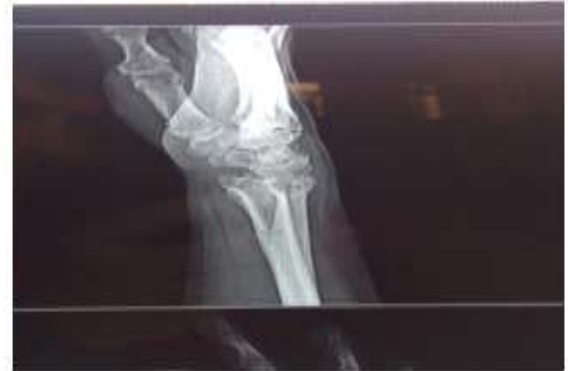

Fig5. X ray after fixator removal 
Fig 6. Movement at 4 months



- Flexion

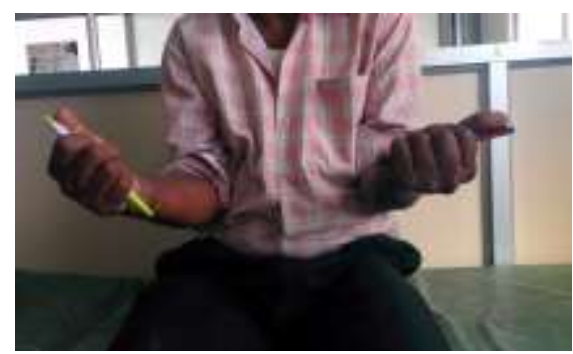

c. Pronation



e. Radial deviation

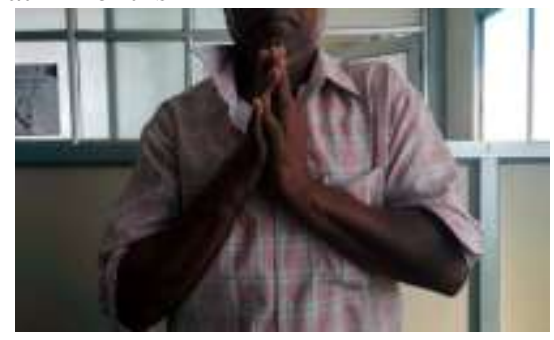

b. Extension

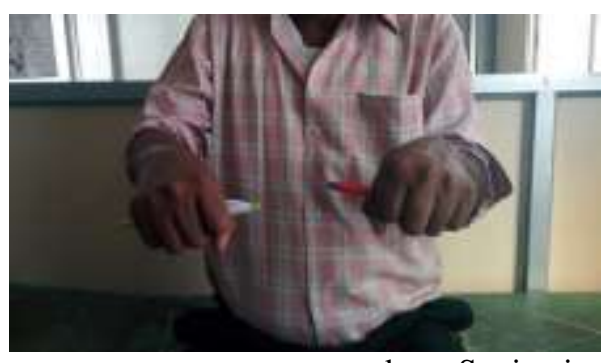

d. Supination

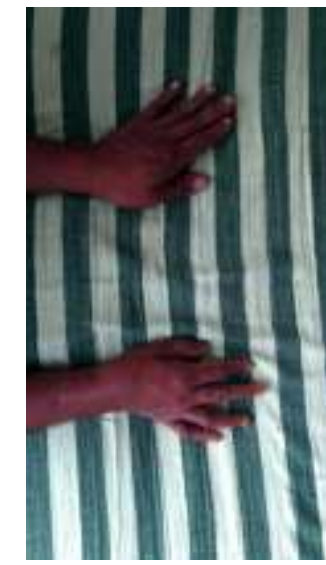

f. Ulnar deviation

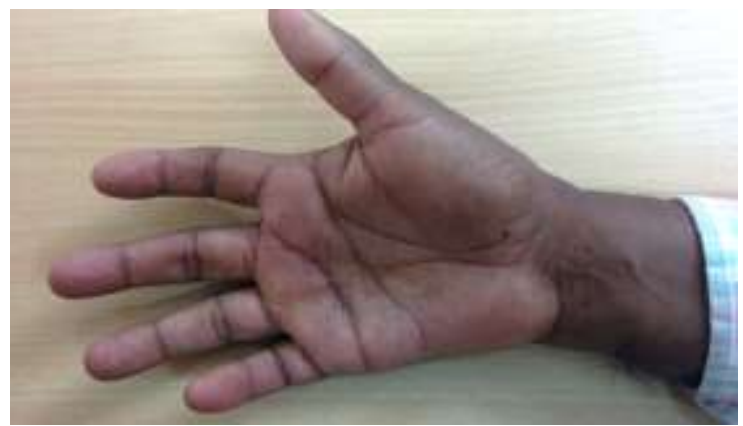

Fig 7. Appearance of the hand at 4 months follow up.

\section{References}

- Girard J, Cassagnaud X, Maynou C, Bachour F, Prodhomme G, Mestdagh H. Radiocarpal dislocation: twelve cases and a review of the literature [in French]. Rev Chir Orthop Reparatrice Appar Mot. 2004; 90(5):426-433

- $\quad$ Dunn AW. Fractures and dislocations of the carpus. Surg Clin North Am. 1972; 52(6):1513-1538.

- Hardy P, Welby F, Stromboni M, Blin JL, Lortat-Jacob A, Benoit J. Wrist arthroscopy and dislocation of the radiocarpal joint without fracture. Arthroscopy. 1999; 15(7):779-783.

- Wilson CJ, Aragon AB, Smith AC. Ulnar nerve palsy following closed radiocarpal fracture-dislocation. Am J Orthop (Belle Mead NJ). 2008; 37(8):E138-E140.

- $\quad$ Ilyas AM, Williamson C, Mudgal CS. Radiocarpal dislocation: is it a rare injury?J Hand Surg Eur Vol. 2011; $36(2): 164$. 